\title{
Clinical features and cognitive sequelae in COVID-19: a retrospective study on $N=152$ patients
}

\author{
Marina Rita Manera ${ }^{1}$ - Elena Fiabane ${ }^{2} \cdot$ Debora Pain $^{3} \cdot$ Edoardo Nicolò Aiello $^{4}\left(\mathbb{D} \cdot\right.$ Alice Radici $^{3} \cdot$ Marcella Ottonello $^{2}$. \\ Mariacristina Padovani ${ }^{1} \cdot$ Barbara Ann Wilson $^{5} \cdot$ Jessica Fish $^{6} \cdot$ Caterina Pistarini $^{7}$
}

Received: 11 October 2021 / Accepted: 11 November 2021 / Published online: 15 November 2021

(c) Fondazione Società Italiana di Neurologia 2021, corrected publication 2021

\begin{abstract}
Background The novel human coronavirus (SARS-CoV-2) shows neurotropism and systemically affects the central nervous system (CNS). Cognitive deficits have been indeed reported as both short- and long-term sequelae of SARS-CoV-2 infection. However, the association between these disturbances and background/disease-related clinical features remains elusive. This work aimed at exploring how post-infective cognitive status relates to clinical/treatment outcomes by controlling for premorbid/current risk factors for cognitive deficits.

Methods Cognitive measures (Mini-Mental State Examination, MMSE) of N=152 COVID-19 patient were retrospectively assessed in relation to disease severity, intensive care unit (ICU) admission, steroidal treatment, and occurrence of other viral/bacterial infections by controlling for remote/recent/COVID-19-related risk factors for cognitive deficits (at-risk vs. not-at-risk: Neuro+ vs. Neuro-).

Results Descriptively, impaired MMSE performances were highly prevalent in mild-to-moderate patients (26.3\%). ICUadmitted patients made less errors $(p=.021)$ on the MMSE than those not admitted when partialling out risk factors and age - the latter negatively influencing performances. When addressing Neuro- patients only, steroidal treatment appears to improve MMSE scores among those suffering from other infections $(p=.025)$.

Discussion Cognitive sequelae of COVID-19 are likely to arise from a complex interplay between background/clinical premorbid features and disease-related/interventional procedures and outcomes. Mild-to-moderate patients requiring assistive ventilation who however are not admitted to an ICU are more likely to suffer from cognitive deficits—-despite their etiology remaining elusive.
\end{abstract}

Keywords SARS-CoV-2 $\cdot$ COVID-19 $\cdot$ Neuropsychology $\cdot$ ICU $\cdot$ Steroid $\cdot$ Premorbid

\section{Introduction}

Central nervous system (CNS) involvement has been acknowledged in patients infected with the novel human coronavirus (SARS-CoV-2)—due to both its neurotropic/ neuroinvasive properties and inflammatory processes/secondary systemic disorders $[8,24]$.

Marina Rita Manera, Elena Fiabane, and Debora Pain equally contributed to the paper.

Edoardo Nicolò Aiello

e.aiello5@campus.unimib.it

Extended author information available on the last page of the article
Cognitive deficits within have been indeed reported and postulated as both short- and long-term sequelae of the disease caused by SARS-CoV-2 (COVID-19) [7, 18, 19].

Most studies suggested deficits in memory, executive functioning, and attention [5, 13, 18, 22, 23]. Furthermore, results from previous pandemics of acute respiratory illness (e.g., middle-east respiratory syndrome) and existing knowledge of neurological outcomes in pulmonary disorders suggested that neuropsychological sequelae are to be expected in patients with COVID-19 [9]. Coronavirus infections are indeed believed to increase and thereby extending the risk of post-infection cognitive dysfunction and accelerating neurodegenerative processes [20].

Pistarini et al. [18] found a high prevalence of cognitive impairments in both COVID-19 and post-COVID-19 patients as assessed by a I-level, global cognition test 
(Montreal Cognitive Assessment). By contrast, a recent 4-month follow-up study [25] investigated cognitive impairments after SARS-CoV-2 infection in a group of mild-moderate post-COVID-19 patients and found no differences compared to non-COVID-19 cases.

It is thus currently debated whether cognitive impairment actually represents a SARS-CoV-2-specific complication or it is secondary to extra-CNS disorders-e.g., systemic inflammation [26].

Moreover, certain issues remain open as to the association between cognitive sequelae and both disease-related and background clinical variables. First, it is challenging to assess post-infective cognitive status by controlling for possibly intervening premorbid conditions/disease-related complications. Second, intensive care unit (ICU) admission has been reported to counterintuitively represent a protective factor toward cognitive outcomes [5]. Moreover, the relation between cognitive dysfunctions and possible iatrogenic effects of steroidal treatment is still poorly understood [12].

The present study thus aimed at investigating how cognitive outcomes relate to clinical/treatment features in COVID19 patients by taking into account premorbid/disease-related clinical features possibly affecting cognition.

\section{Methods}

\section{Materials}

Data from $N=152$ post-infectious SARS-CoV-2 patients referred to either sub-acute or specialist rehabilitation units of Istituti Clinici Scientifici Maugeri located in Northern Italy, between May 2020 and May 2021, were retrospectively collected (see Table 1). The study was approved by the local Ethical Committee (Approval Number: 2470, 8 September 2020).

All patients had been administered the Mini-Mental State Examination (MMSE) [17]—the most commonly used tool for screening cognitive impairment and consists of a brief (5-10) 30-point scale. The presence of cognitive impairment was defined by a total score $<23.80$ adjusted for age and education in the Italian population [17]. .

Furthermore, information regarding neurological, psychiatric, and general medical history were retrieved, along with data regarding the clinical manifestations of COVID-19. A classification according to disease severity was performed: asymptomatic; mildly symptomatic; mild-to-moderate: requiring $\mathrm{O}_{2}$ therapy but not ventilation; moderate-to-severe: requiring either non-invasive ventilation or admitted to an ICU.

Furthermore, patients were sub-divided into those who had either remote, recent, or COVID-19-related conditions possibly affecting cognitive functioning (Neuro+) and those who did not (Neuro-). The Neuro+ group included patients with (a) neurological diseases (e.g., Parkinson's disease, stroke); (b) severe psychiatric disorders (e.g., depression, post-traumatic stress-disorder); (c) severe internal conditions (e.g., atrial fibrillation); and (d) at least 3 risk factors for NP impairment (e.g., type-II diabetes, arterial hypertension, and chronic obstructive pulmonary disease). This group however did not encompass patients that suffered from acute respiratory distress syndrome (ARDS)/respiratory insufficiency (requiring or not assistive ventilation) or were admitted to an ICU due to COVID-19. This expedient was implemented in order to rule out possible overlapping co-occurrences with the ICU/Severity factors. As to the inclusion criteria of Neuro-, they did not present with the aforementioned risk factors for cognitive decline.

Two independent authors performed this categorization blinded to both each other's decision and patients' psychometric outcomes; disagreements were solved by discussion with a third independent author. According to this grouping, 103 patients were classified as Neuro+ and 49 as Neuro-.

\section{Statistical analyses}

Normality checks were performed by assessing skewness and kurtosis values [16].

According to data distribution, either linear or generalized linear models [1] were implemented for assessing predictions of interest. Associations between continuous variables were tested via either Pearson's or Spearman's coefficient.

Group (Neuro+ vs. Neuro-) was partialled out in each model in order to control for premorbid/disease-related confounders. As Neuro+ and Neuro- patients were comparable for education $(t(150)=.63 ; p=.366)$ but not for age $(t(150)=$ -2.05 ; $p=.042$; Neuro+: $M=68.5, S D=13.7$; Neuro-: $M=63.8, S D=11.5)$, the latter was entered as a covariate within models including Group.

ICU (admitted vs. not admitted), Steroids (treated vs. not treated with steroids), Infection (occurrence vs. absence of a bacterial/viral infection during COVID-19), and Severity (mild, recoded by merging the first two original levels into one vs. mild-to-moderate vs. moderate-to-severe) effects were tested on both the MMSE and its sub-scores. Domainspecific scales were defined as follows: spatial and temporal orientation (0-10); immediate and delayed recall (0-6); attention (0-5); language (0-8); constructional praxis (0-1). Within each implemented model, interactions between target (e.g., ICU) and control (i.e., Group and Age) variables, as well as between control variables themselves, were not tested.

Bonferroni correction for multiple comparisons was applied if appropriate.

Analyses were performed via SPSS 27 [14] and jamovi $1.6[21]$. 
Table 1 Participants' background, clinical and psychometric measures

\begin{tabular}{|c|c|c|c|c|c|}
\hline Domain & & Outcome & & & \\
\hline Backgrou & & & & & \\
\hline & & $N$ & 152 & & \\
\hline & & Age (years) & $67 \pm 13.2(18-93)$ & & \\
\hline & & Sex (male/female) & $101 / 51$ & & \\
\hline & & Education (years) & $10.6 \pm 3.9(2-19)$ & & \\
\hline Clinical & & & & & \\
\hline & & Disease duration (days) & $43.4 \pm 25.6(2-129)$ & & \\
\hline & & Time from onset (days) & $84 \pm 65.6(7-422)$ & & \\
\hline & Severity & Asymptomatic & $8.6 \%$ & & \\
\hline & & Mildly symptomatic & $15.1 \%$ & & \\
\hline & & Mild-to-moderate & $25 \%$ & & \\
\hline & & Moderate-to-severe & $51.3 \%$ & & \\
\hline & & ICU & $48.7 \%$ & & \\
\hline & & Steroids & $38 \%$ & & \\
\hline & & Infection & $31.1 \%$ & & \\
\hline & Comorbi & & & & \\
\hline & & & Remote & Recent & $\begin{array}{c}\text { COVID- } \\
\text { 19-re- } \\
\text { lated }\end{array}$ \\
\hline & & Neurological & $30.6 \%$ & $15.1 \%$ & $28.9 \%$ \\
\hline & & Psychiatric & $33.3 \%$ & $5.9 \%$ & $3.3 \%$ \\
\hline & & Cardiac & $59.2 \%$ & $3.3 \%$ & $7.9 \%$ \\
\hline & & Pulmonary & $12.9 \%$ & $2 \%$ & $19.1 \%$ \\
\hline & & Infective & $5.4 \%$ & $.7 \%$ & $5.9 \%$ \\
\hline & & Metabolic & $23.1 \%$ & - & $2 \%$ \\
\hline Psychom & & & & & \\
\hline MMSE & & Total & $27.3 \pm 3.1(15-30)$ & & \\
\hline & & Temporal orientation & $4.5 \pm .9(1-5)$ & & \\
\hline & & Spatial orientation & $4.6 \pm .7(2-5)$ & & \\
\hline & & Immediate recall & $3 \pm .2(1-3)$ & & \\
\hline & & Attention & $4.4 \pm 1.3(0-5)$ & & \\
\hline & & Delayed recall & $2.3 \pm .9(0-3)$ & & \\
\hline & & Language & $7.8 \pm .6(4-9)$ & & \\
\hline & & Constructional praxis & $.8 \pm .4(0-1)$ & & \\
\hline
\end{tabular}

MMSE, Mini-Mental State Examination; ICU, intensive care unit; COVID-19, coronavirus disease 2019

\section{Results}

Overall prevalence of cognitive deficits as assessed via the MMSE was $12.5 \%$. Table 2 displays prevalence estimates sub-divided according to target factors. Below-cutoff MMSE percentage was visibly higher in Neuro+ $(16.5 \%)$ vs. Neuro- $(4.1 \%)$ patients. Moreover, within severity degrees, impaired MMSE performances were notably more frequent for mild-to-moderate (26.3\%). Finally, a trend toward a lower prevalence of defective MMSE scores was detected in ICU-admitted patients (19.2\%)—when descriptively compared to those not admitted (5.4\%).

When testing the association between MMSE scores and disease duration/time from onset to evaluation separately for the four severity sub-groups, no significant coefficients arose at $\alpha_{\text {adjusted }}=.05 / 4=.013$.

Both MMSE total and sub-scores were heavily leftskewed and overdispersed. Therefore, predictions on the MMSE were initially run via negative binomial regressions, by addressing the number of errors (subtracting the score to its maximum achievable) as the outcome [1].

When individually testing target factors on MMSE total errors with Group and Age partialled out, a significant effect of $I C U$ arose $\left(\chi^{2}(1)=5.3 ; p=.021\right)$-with ICU-admitted patients $(M=1.72 ; S E=.24)$ making less errors than those not admitted $(M=2.73 ; S E=.38)$; by contrast, neither Severity $\left(\chi^{2}(3)=2.07 ; p=.356\right)$ nor Steroids $\left(\chi^{2}(1)=.49\right.$; $p=.485)$ nor Infection $\left(\chi^{2}(1)=.8 ; p=.372\right)$ yielded significance. Notably, age negatively influenced the performance 
Table 2 Below-cutoff scores on the MMSE according to diseaserelated variable

\begin{tabular}{lll}
\hline & & $<23.8 \dagger$ \\
\hline Severity & Asymptomatic & \\
& Mildly symptomatic & $7.7 \%$ \\
& Mild-to-moderate & $13 \%$ \\
& Moderate-to-severe & $26.3 \%$ \\
& Neuro+ & $6.4 \%$ \\
& Neuro- & $16.5 \%$ \\
ICU & Admitted & $4.1 \%$ \\
& Not admitted & $5.4 \%$ \\
Steroids & Yes & $19.2 \%$ \\
& No & $13.2 \%$ \\
Infections & Yes & $12.9 \%$ \\
& No & $8.5 \%$ \\
& & $14.4 \%$ \\
\hline
\end{tabular}

MMSE, Mini-Mental State Examination; Neuro+/-, patients with/ without remote/recent/disease-related comorbidities possibly affecting cognition. †Cutoff from Measso et al. [17]

in all the above models $(p \leq .002)$, whereas Group never showed significance $(.052 \leq p \leq .28)$. Consistent results were detected when building a model encompassing ICU, Infection, and Steroids along with their interactions: ICU and age were predictive per se $\left(\chi^{2}(1)=5.5 ; p=.019\right.$ and $\chi^{2}(1)=4.7 ; p=.029$, respectively), whereas no other main $(.395 \leq p \leq .75)$ or interactive $(.1 \leq p \leq .93)$ terms were significant.

As being the only significant target factor in previous models, ICU was further tested on MMSE Orientation, Attention, Memory and Language errors by controlling for Group and age. The same model was instead tested on constructional praxis via a logistic regression. ICU was not found to affect performances on any of the sub-scales $(.112 \leq p \leq .311)$. However, ICU admission predicted $\left(\chi^{2}(1)=4.4 ; p=.036\right)$ a higher probability $(M=.88 ; S E=.04)$ of responding correctly to constructional praxis itemwhen compared to non-admission $(M=.72 ; S E=.06)$.

Factors of interest were then further tested on Neuro- patients' MMSE scores only $(N=49)$ by controlling for age and education. As both normality ( $W=.957$; $p=.38)$ and homoscedasticity $(F(7,16)=1.5 ; p=.435)$ assumptions for residuals were met, a linear model was run-which encompassed all possible between-factor interactions. No significant terms arose with the exception of a two-way Steroids $\times$ Infection interaction $(F(1,14)=6.3$; $p=.025 ; \eta^{2}=.31$ ) - whose post hoc, Bonferroni-corrected decomposition revealed that, among patients suffering from infections, those treated with steroids performed significantly $(t(14)=-3.86 ; p=.01)$ better $(M=29.1 ; S E=.64)$ than those not treated with steroids $(M=25.5 ; S E=.67)$.

\section{Discussion}

This work sheds further light on the association between cognitive sequelae of SARS-CoV-2 infection and premorbid/disease-related clinical variables [7].

With respect to the protective role of ICU admission on cognitive functions, the present results are in line with the report by [5]. It can thus be hypothesized that patients presenting with ARDS/respiratory insufficiency who underwent intensive cares might have suffered less from cerebral hypoxia than those treated with non-invasive ventilation [5]—despite these treatments being more aggressive.

Furthermore, ICU admission being shown to affect global cognition but not specific instrumental domains further supports the notion that COVID-19-related cognitive deficits are likely to reflect a decrease in general cognitive efficiency-which is typical of critical illnesses also affecting the CNS [15].

It is moreover worth mentioning that the trend toward a poorer cognitive outcome in mild-to-moderate patients when compared to both mild and moderate-to-severe ones also appears to mirror Alemanno et al.'s [5] findings.

The present work does not provide overall conclusive evidence regarding the association between cognitive outcomes and steroidal treatment in COVID-19 patients [12]. This might have been due to missing values as far as whether patients have been treated with steroids (information not available for $N=52$ patient).

However, when selectively assessing patients judged as not at risk for cognitive impairment, steroids appeared to improve cognitive outcomes when infections occurred during the disease course. Therefore, although steroidal interventions have been postulated as possibly iatrogenic on cerebral functions [12], they might be beneficial to cognitive outcomes when other inflammatory processes co-occur with COVID-19.

As for background outcomes, findings here reported strongly support the role of advanced age as a risk factor for a worse cognitive outcome in post-infective SARSCoV-2 patients [6]. Moreover, although no strong inferential evidence emerged, a descriptive trend toward a higher prevalence of cognitive dysfunction in already-at-risk COVID-19-recovered patients could be noted [6].

A limitation of this report is represented by the fact that only the MMSE has been addressed as a cognitive measure, this possibly leading to an underestimation of the prevalence of COVID-19-related cognitive aftermaths. Indeed, it has been suggested that other screeners, such as the Montreal Cognitive Assessment (MoCA) [3] and the Frontal Assessment Battery [2], may be more appropriate for detecting such dysfunctions-possibly due to the an higher sensitivity $[4,10]$. 
In conclusion, cognitive sequelae of COVID-19 are likely to arise from a complex interplay between background/clinical premorbid features and disease-related/interventional procedures and outcomes. Mild-to-moderate patients requiring assistive ventilation who however are not admitted to an ICU are more likely to suffer from cognitive deficitsdespite their etiology remaining elusive. Further investigations are thus needed, also focusing on the longitudinal interplay of cognition and clinical features [11].

Acknowledgements The authors are grateful to all participants. The authors would like to thank Dr. Sharon Brambilla for her precious help to data collection.

\section{Declarations}

This study was conducted in accordance with the Declaration of Helsinki.

\section{Conflict of interest None}

Ethical approval Patients provided their informed consent. This study received ethical approval.

\section{References}

1. Aiello EN, Depaoli EG, Gallucci M (2020) Usability of the negative binomial model for analyzing ceiling and highly-inter-individually-variable cognitive data. Neurol Sci 41:S273-S274. https:// doi.org/10.1007/s10072-020-04753-3

2. Aiello EN, Esposito A, Gramegna C, Gazzaniga V, Zago S, Difonzo T, ... \& Bolognini N (2021a). The Frontal Assessment Battery (FAB) and its sub-scales: validation and updated normative data in an Italian population sample. Neurol Sci, 1-6

3. Aiello EN, Fiabane E, Manera MR, Radici A, Grossi F, Ottonello M, Pain D, Pistarini C (2021b) Screening for cognitive sequelae of SARS-CoV-2 infection: a comparison between the Mini-Mental State Examination (MMSE) and the Montreal Cognitive Assessment (MoCA). Neurol Sci:1-4

4. Aiello EN, Gramegna C, Esposito A, Gazzaniga V, Zago S, Difonzo T, ... \& Bolognini N (2021c) The Montreal Cognitive Assessment (MoCA): updated norms and psychometric insights into adaptive testing from healthy individuals in Northern Italy. Aging Clinical and Experimental Research, 1-8

5. Alemanno F, Houdayer E, Parma A, Spina A, Del Forno A, Scatolini A, Angelone S, Brugliera L, Tettamanti A, Beretta L, Iannaccone S (2021) COVID-19 cognitive deficits after respiratory assistance in the subacute phase: a COVID-rehabilitation unit experience. PLoS One 16:e0246590

6. Almeria M, Cejudo JC, Sotoca J, Deus J, Krupinski J (2020) Cognitive profile following COVID-19 infection: clinical predictors leading to neuropsychological impairment. Brain, Behavior, \& Immunity-Health 9:100163

7. Alnefeesi Y, Siegel A, Lui LM, Teopiz KM, Ho R, Lee Y, Nasri F, Gill H, Lin K, Cao B, Rosenblat JD (2021) Impact of SARSCoV-2 infection on cognitive function: a systematic review. Front Psych 11:1629

8. Baig AM, Khaleeq A, Ali U, Syeda H (2020) Evidence of the COVID-19 virus targeting the CNS: tissue distribution, host-virus interaction, and proposed neurotropic mechanisms. ACS Chem Neurosci 11:995-998

9. Bailey EK, Steward KA, VandenBussche Jantz AB, Kamper JE, Mahoney EJ, Duchnick JJ (2021) Neuropsychology of COVID-19: anticipated cognitive and mental health outcomes. Neuropsychology $35: 335$

10. Beaud V, Crottaz-Herbette S, Dunet V, Vaucher J, Bernard-Valnet R, Du Pasquier R, Clarke S (2021) Pattern of cognitive deficits in severe COVID-19. J Neurol Neurosurg Psychiatry 92:567-568

11. Blazhenets G, Schröter N, Bormann T, Thurow J, Wagner D, Frings $L$ et al (2021) Slow but evident recovery from neocortical dysfunction and cognitive impairment in a series of chronic COVID-19 patients. J Nucl Med

12. Ghasemiyeh P, Borhani-Haghighi A, Karimzadeh I, MohammadiSamani S, Vazin A, Safari A, Qureshi AI (2020) Major neurologic adverse drug reactions, potential drug-drug interactions and pharmacokinetic aspects of drugs used in COVID-19 patients with stroke: a narrative review. Ther Clin Risk Manag 16:595

13. Helms J, Kremer S, Merdji H, Clere-Jehl R, Schenck M, Kummerlen $\mathrm{C}$ et al (2020) 415 Neurologic features in severe SARS-CoV-2 infection. N Engl J Med 382:2268-2270

14. IBM Corp (2020) IBM SPSS Statistics for Windows, Version 27.0. IBM Corp, Armonk, NY

15. Jaywant, A., Vanderlind, W. M., Alexopoulos, G. S., Fridman, C. B., Perlis, R. H., Gunning, F. M. (2021). Frequency and profile of objective cognitive deficits in hospitalized patients recovering from COVID-19. Neuropsychopharmacology, 1-6.

16. Kim HY (2013) Statistical notes for clinical researchers: assessing normal distribution (2) using skewness and kurtosis. Restorative Dentistry \& Endodontics 38:52-54

17. Measso G, Cavarzeran F, Zappala G, Lebowitz BD, Crook TH, Pirozzolo FJ, Amaducci LA, Massari D, Grigoletto F (1993) The Mini-Mental State Examination: normative study of an Italian random sample. Dev Neuropsychol 9:77-85

18. Pistarini, C., Fiabane, E., Houdayer, E., Vassallo, C., Manera, M.R., Alemanno, F. (2021) Cognitive and emotional disturbances due to COVID-19: an exploratory study in the rehabilitation setting. Frontiers in Neurology, 12, 643-646.

19. Rabinovitz B, Jaywant A, Fridman CB (2020) Neuropsychological functioning in severe acute respiratory disorders caused by the coronavirus: implications for the current COVID-19 pandemic. Clin Neuropsychol 34:1453-1479

20. Ritchie K, Chan D, Watermeyer T (2020) The cognitive consequences of the COVID-19 epidemic: collateral damage? Brain Commun. 2:fcaa069. https://doi.org/10.1093/braincomms/fcaa0 69

21. The jamovi project (2021). jamovi. (Version 1.6) [Computer Software]. Retrieved from https://www.jamovi.org.

22. Wilson BA, Betteridge S, Fish J (2020) Neuropsychological consequences of COVID-19. Neuropsychol Rehab 30:1625-1628

23. Zhou H, Lu S, Chen J, Wei N, Wang D, Lyu H et al (2020) The landscape of cognitive function in recovered COVID-19 patients. J Psychiatr Res 129:98-102

24. Zubair AS, McAlpine LS, Gardin T, Farhadian S, Kuruvilla DE, Spudich S (2020) Neuropathogenesis and neurologic manifestations of the coronaviruses in the age of coronavirus disease 2019: a review. JAMA Neurology 77:1018-1027

25. Mattioli F, Stampatori C, Righetti F, Sala E, Tomasi C, De Palma G (2021) Neurological and cognitive sequelae of Covid-19: a four month follow-up. J Neurol 268:4433-4428

26. Stracciari A, Bottini G, Guarino M, Magni E, Pantoni L (2021) Cognitive and behavioral manifestations in SARS-CoV-2 infection: not specific or distinctive features?. Neurol Sci 1-9

Publisher's note Springer Nature remains neutral with regard to jurisdictional claims in published maps and institutional affiliations. 


\title{
Authors and Affiliations
}

\section{Marina Rita Manera ${ }^{1}$ - Elena Fiabane ${ }^{2} \cdot$ Debora Pain $^{3} \cdot$ Edoardo Nicolò Aiello $^{4}\left(\mathbb{0} \cdot\right.$ Alice Radici $^{3} \cdot$ Marcella Ottonello $^{2}$. Mariacristina Padovani ${ }^{1}$. Barbara Ann Wilson ${ }^{5}$. Jessica Fish ${ }^{6}$. Caterina Pistarini ${ }^{7}$}

\author{
Marina Rita Manera \\ marina.manera@icsmaugeri.it \\ Elena Fiabane \\ elenamaria.fiabane@icsmaugeri.it \\ Debora Pain \\ debora.pain@icsmaugeri.it \\ Alice Radici \\ alice.radici@icsmaugeri.it \\ Marcella Ottonello \\ marcella.ottonello@icsmaugeri.it \\ Mariacristina Padovani \\ mariacristina.padovani@icsmaugeri.it \\ Barbara Ann Wilson \\ barbara.wilson00@gmail.com \\ Jessica Fish \\ Jessica.Fish@glasgow.ac.uk \\ Caterina Pistarini \\ caterina.pistarini@icsmaugeri.it
}

1 Istituti Clinici Scientifici Maugeri, IRCCS, Psychology Unit of Pavia Institute, Pavia, Italy

2 Istituti Clinici Scientifici Maugeri, Department of Physical and Rehabilitation Medicine of Genova Nervi Institute, Genoa, Italy

3 Istituti Clinici Scientifici Maugeri, IRCCS, Neurorehabilitation Department of Milano Institute, Milan, Italy

4 PhD Program in Neuroscience, School of Medicine and Surgery, University of Milano-Bicocca, Monza, Italy

5 Department of Clinical Neuropsychology \& Clinical Health Psychology, St George's University, London, UK

6 Institute of Health and Wellbeing, University Of Glasgow, Glasgow, UK

7 Istituti Clinici Scientifici Maugeri, IRCCS, Department of Neurorehabilitation of Pavia Institute, Pavia, Italy 